\title{
Una aproximación escéptica a la relación entre epistemología y derecho
}

\author{
Mana iñiyu aśhuykuy yaćhayka \\ kamachikuypula tinkuyninćhu
}

\section{Aregiteengagantsi ora kengagantsi aike otigëgotagantsi}

\author{
Recepción: 23 julio 2020 Aprobación: 29 septiembre 2020
}

Enlil Iván Herrera Pérez

Nacionalidad: Peruana

Universidad Privada de Tacna

Correo: eiherrera@upt.pe

ORCID: https://orcid.org/0000-0002-0050-2882

\section{Resumen}

En el presente artículo el autor presenta los principales conceptos relativos a la epistemología, la ciencia y la búsqueda de la verdad desde la óptica de Bunge, Tarski, Popper y Kuhn. Conceptos que vincula con una noción instrumental del derecho con el objetivo de responder a la pregunta de si existe o no una relación entre epistemología y Derecho, y acerca de qué tipo de relación sería tal. Sobre esa base, y con un criterio escéptico concluye destacando la relevancia de la ciencia y el derecho.

\section{Palabras clave:}

Derecho, epistemología, ciencia, verdad, positivismo jurídico.

\section{Lisichiku limaykuna:}

Kamchiy, yaćhana, yaćhay, rasunka, śhaykupaku kamachiy.

\section{Nibarintsi katingaro:} kantëgotagantsi, kengagantsi, aisoritagantsi, kaninaengaro kantagansi

\section{Datos del autor}

Enlil Iván Herrera Pérez es investigador en filosofía jurídica, filosofía política, teoría general del derecho y derecho privado. Maestro en Derecho por la Universidad Privada de Tacna, Perú. Y actualmente cursa el programa de Doctorado en Derecho en la misma casa de estudios. 


\section{A Skeptical Approach to the Relationship Between Epistemology and Law}

\begin{abstract}
In this article the author presents the main concepts related to epistemology, science and the search for truth from the perspectives of Bunge, Tarski, Popper and Kuhn. Concepts that relates to an instrumental notion of law. All this with the aim of answering the question of whether or not there is a relationship between epistemology and law, and about what kind of relationship would exist. On this basis, and with a skeptical approach, it concludes by highlighting the relevance of science and law.
\end{abstract}

\section{Keywords}

Law, epistemology, science, truth, legal positivism.

\section{Uma aproximação cética à relação entre epistemologia e direito}

\section{Resumo}

Neste artigo o autor apresenta os principais conceitos relacionados relativos à epistemologia, a ciência e à busca da verdade na perspectiva de Bunge, Tarski, Popper e Kuhn. Conceitos que se vinculam a uma noção instrumental do Direito com o objetivo de responder à questão de se existe ou não uma relação entre epistemologia e Direito, e sobre que tipo de relação seria essa. Com base nisso, e com um critério cético, conclui-se destacando a relevância da ciência e do Direito.

\section{Palavras-chave:}

Direito, epistemologia, ciência, verdade, positivismo jurídico. 


\section{Epistemología y Derecho: a modo de introducción}

Sin lugar a dudas, la búsqueda de conocimiento ha sido una actividad constante a lo largo de la historia humana -aunque son igual de innegables los episodios de represión ante tal actividad. Y no una búsqueda de mera opinión o dogma, sino de un conocimiento de validez reconocible -respecto a su proceso de adquisición como también al resultado obtenible. Partiendo de ello es posible advertir que el problema es exactamente la determinación de dicha validez, problema del cual se ocupa la epistemología tal como es entendida contemporáneamente (Ceberio \& Watzlawick, 1998).

Por otro lado, en el siglo XX se destacaría una preocupación similar dentro del campo del Derecho, esta vez buscando argumentar fundamentos de validez para un Ilamado conocimiento científico del Derecho (Kelsen, 1982), como también para un denominado discurso racional del Derecho (Alexy, 1991) -en ambos casos, buscando argumentar la racionalidad interna de lo jurídico.

Partiendo de la aparente vinculación de disciplinas, cabe realizar diversas preguntas, en primer lugar, acerca de si existe o no una relación entre epistemología y Derecho; de si es posible una epistemología jurídica; o -de ser el caso- de qué tipo de relación puede establecerse, entonces, entre epistemología y Derecho. Preguntas que se justifican ante el riesgo de extrapolación de conceptos y métodos que podrían culminar en una deformación de ambas disciplinas.

Para responder a dichas interrogantes, en el presente analizaremos cuatro puntos de vista propuestos respectivamente bajo la concepción de ciencia argumentada por Mario Bunge; la concepción semántica de la verdad en Alfred Tarski; la verdad según el falsacionismo de Karl Popper; y bajo el desarrollo de la ciencia en un contexto de crisis y revolución en Thomas Kuhn. Para finalmente realizar un balance general de lo comentado a modo de conclusión, buscando sintetizar las respuestas a las inquietudes planteadas conforme a lo discutido desde cada uno de los cuatro puntos de vista abordados.

\section{El Derecho en Bunge}

Mario Bunge ha sido uno de los principales referentes de la ciencia, ocupándose en forma sumamente clara -y crítica- de distinguir las ciencias en sentido estricto (Bunge, 2014) de las seudociencias (Bunge, 2011b). Para Bunge (2014) lo que permite identificar cuándo estamos frente a un conocimiento científico, y cuándo no, son las características de tal conocimiento: su facticidad, su trascendencia a los hechos, el corte analítico, la especialización, la comunicabilidad por la precisión del conocimiento, la verificabilidad del mismo en la realidad, el método en la investigación científica que lo produce, su sistematización, la generalidad, y su legalidad en términos naturales o necesarios, su posibilidad explicativa que no se reduce a lo causal, la predictibilidad, su apertura, y utilidad.

Características que además deben ser identificables, puesto que la finalidad última de la ciencia es ser una herramienta para "domar la naturaleza y remodelar la sociedad" (Bunge, 2014, p. 24).

Es así que, en el pensamiento de Bunge (2011a), el Derecho no podría ser considerado como una ciencia. Para Bunge, el Derecho es "un sistema de normas (en particular leyes), meta-normas [que podríamos llamar principios], opiniones expertas, precedentes legales y 
debates sobre el comportamiento social permisible, prohibido y obligatorio" (Bunge, 2011, p. 384). Y aunque el contexto de este concepto de Bunge sea el campo anglosajón, el propio Bunge remite a una lectura de Kelsen (1982) o Raz (1986) para una concepción del Derecho más restringida -como sistema normativo.

Y ciertamente, aunque Kelsen pueda Ilamar "ciencia” al Derecho, define a este último como una ciencia sui géneris en su Teoría Pura del Derecho (1982). Pues, a pesar de definirse un objeto y un método en todas las ciencias sociales -incluyendo al Derecho- mientras en cualquier otra ciencia social pueda percibirse el establecimiento de leyes científicas causales y de conclusión necesarias, en el Derecho las leyes [o, mejor dicho, normas] son establecidas por imputación y las conclusiones determinadas son deónticas. Las consecuencias frente a un estímulo determinado en las ciencias tradicionales se producen en forma necesaria, sin ser relevante la búsqueda o no de dichas consecuencias, simplemente basta la ocurrencia del estímulo. En cambio, en el Derecho previamente se requiere de una voluntad institucional que establezca, o impute, una consecuencia jurídica frente a un hecho determinado. Esta consecuencia jurídica, a diferencia de la anterior, no se produce en forma necesaria, sino que existe un mandato de que debería producirse - puesto que mucho dependerá de diversas otras variables, sobre todo de aquella autonomía institucionalizada.

La racionalidad interna del Derecho, para Kelsen (1982), dependerá de su consistencia y completitud como sistema. Sistema que existe, según Kelsen tomando las tesis primigeniamente planteadas por Adolf Merkl (Paulson, 2000), por vincular sus elementos [normas jurídicas] a través de relaciones genéticas o dinámicas, relaciones que se explican bajo criterios de deducibilidad y legalidad, respectivamente. Sin embargo, pese a argumentarse una racionalidad propia, un sistema jurídico no se forma sino sustancialmente bajo autonomía institucional, esto es: la voluntad de quienes se les reconoce competencia (Hart, 2009).

Así, pese a haberse argumentado dicha racionalidad en el Derecho, ello no sería suficiente para atribuirle la condición de ciencia al Derecho, al menos según la teoría de Bunge (2011a; 2011b; 2014).

Sobre esa base ¿sería posible hablar de una epistemología del Derecho? En Bunge, esta pregunta sería fácilmente respondida, puesto que la primera condición que debe satisfacer la concepción de epistemología para ser útil es considerar que esta "concierne a la ciencia propiamente dicha, no a la imagen pueril y a veces hasta caricaturesca tomada de libros de texto elementales" (Bunge, 1976, p. 5). Esta condición excluye en forma liminar la posiblidad de una epistemología del Derecho al menos, nuevamente, según la teoría de Bunge.

¿Ello significaría que no puede aportarse al Derecho desde la epistemología, o que no puede aportarse a la epistemología desde el Derecho? No necesariamente. Aunque sí es posible aifrmar que entre epistemología y Derecho no existe una relación directa ni de pertenencia. Le relación será, entonces, indirecta y con fines esencialmente instrumentales -condición que si le atribuye Bunge (2011a) al Derecho.

Para Bunge (1976), una filosofía "aceptable" de la ciencia -es decir, una epistemología- es aquella que (a) concierne a la ciencia propiamente dicha, (b) se ocupa de problemas filosóficos presentes en el curso de la inveistigación científica, (c) propone soluciones consistentes en teorías rigurosas o inteligibles, (d) distingue entre ciencia auténtica y seudociencia, y (e) es capaz de criticar así como de sugierir nuevos enfoques. 
El Derecho es instrumento útil para la epistemología a razón de permitir desarrollar medios eficaces para los problemas éticos de la ciencia (Bunge, 1976). Esto en razón de la fuerza institucional que acompaña a las normas válidamente expedidas, así como en razón de su potencial como motivación indirecta (Kelsen, 1982), incentivando o desincentivando la realización de conductas determinadas a través de premios o castigos, sean ex ante o bien ex post. Lo que permite no sólo "un código moral mínimo para la comunidad científica" (Bunge, 1976), sino un código además de moral, efectivo (Kelsen, 1982) y eficiente (Nino, 1992).

De otro lado, la epistemología es un instrumento útil para el Derecho en cuanto a la facticidad de este último en el sentido del elemento asertivo -palabra-a-mundo- que requiere para establecer una dirección de ajuste mundo-a-palabra (Anscombe, 1991). Dicho de otro modo, para representar cómo debería ser el mundo, primero es necesario conocer la realidad de dicho mundo. Este último conocimiento, accesible desde la ciencia. Aunque, claro está, el Derecho siempre agregará el componente valorativo, sea sobre una base normativa (Kelsen, 1982) o sobre una base de justificación valorativa racional de aquello debido (Alexy, 1991). Situación presente tanto en la aplicación como en la formación del Derecho (Raz, 1986).

\section{Tarski y la concepción semántica de la verdad}

La verdad, objeto más elevado de contemplación desde la filosofía griega para muchos pensadores, para algunos un objeto accesible al ser humano, aunque para otros inaccesible e incluso inexistente (Störig, 2012). Objeto, sin duda, de dilema.

Si algún autor contemporáneo, buscó darle contenido a la noción de verdad desde un punto de vista particular, ese es Alfred Tarski. Matemático, lógico y filósofo "de cierta especie" -como se define a sí mismo- se ocupó de abordar los fundamentos de la semántica y de la definición de la verdad (Tarski, 1956). Ello le valió para esbozar una concepción semántica de la verdad (Tarski, 2005) bajo un punto de vista particular producto de su formación como matemático y como lógico.

Para Tarski (2005) una definición satisfactoria de verdad requiere previamente el establecimiento de condiciones de adecuación -desde el punto de vista material- y de corrección -desde el punto de vista formal.

En cuanto al punto de vista material, Tarski parte de una propuesta tanto para la extensión del término verdad como para el significado del mismo término. Así, en cuanto a su extensión, la noción de verdad debe vincularse a un lenguaje específico, aplicando dicha noción a enunciados asertivos, o, como denomina Tarski (2005) "oraciones enunciativas". Y en cuanto al significado del término verdad, expresa dicha concepción de dos modos (Tarski, 2005, pp. 301, 302):

(1) La verdad de una oración consiste en su acuerdo (o correspondencia) con la realidad.

(2) Una oración es verdadera si designa un estado de cosas existente.

El primer modo bajo terminología filosófica moderna, y el segundo de un modo más popular en cuanto al término designa. En ambos casos, el significado alude a la extensión del término verdad, tal como plantea Tarski. La realidad, entonces, será expresada como lenguaje-objeto, y la oración o enunciado como el metalenguaje -es decir, el lenguaje en que se habla de la realidad. 
Sin embargo, Tarski $(1956 ; 2005)$ advierte la insuficiencia de condición material para que por sí sola permita acreditar la correspondencia entre el enunciado y la realidad. Para Tarski la definición de verdad requiere de enunciados de lenguaje perfectamente especificados, por lo que dicho autor opta por el empleo de un lenguaje formal, aporte de los lógicos y matemáticos. La pertinencia de ello, radica en que los llamados lenguajes naturales -aquellos que empleamos en forma cotidiana- adolecen de cierto grado de imprecisión de aquello que se desea transmitir (Hospers, 1984), surgiendo así problemas no sólo de interacción cotidiana, sino incluso de interpretación y aplicación en el Derecho (Hart, 2009).

Entonces, evitando dichos problemas de imprecisión en el lenguaje, a través de enunciados perfectamente especificados, Tarski trata de ver logrado su argumento de que a través de la semántica es posible construir una definición satisfactoria de verdad. Para finalmente (2005) sugerir que dicha propuesta debe verse quizá no como un fin en sí mismo, sino como un medio para la investigación y la búsqueda del conocimiento.

La pregunta que se propone ahora, es la siguiente: ¿qué tanto importa la verdad en el Derecho? Sin duda alguna, la relevancia es indiscutible en cuanto a los presupuestos fácticos para la formación y aplicación del Derecho. Pero, ¿en cuando a los elementos normativos?

Considerando que las formulaciones normativas implican enunciados u oraciones deónticas, estarían excluidas del análisis de verdad, al menos en la teoría de Tarski. Sin embargo, resulta interesante observar el caso de las proposiciones normativas -que sí implican oraciones enunciativas. Para ello, es primeramente necesario diferenciar ambas oraciones.

(1) El concebido es sujeto de derechos en todo cuanto le favorece.

(2) Hay una norma que establece "El concebido es sujeto de derechos en todo cuanto le favorece".

A diferencia de la formulación normativa descrita en el punto (1), que encierra una oración deóntica, de la cual no puede verificarse su verdad o falsedad; el punto (2) es una proposición, que encierra una oración enunciativa, de la cual sí puede verificarse su verdad o falsedad. Es dicha proposición, entonces, el metalenguaje del Derecho -que sería en este caso el lenguaje-objeto en palabras de Tarski (2005)- o, digamos, una representación del mismo (Bulygin, 2018).

Sin embargo, cabe preguntarse ¿el Derecho -como lenguaje-objeto- es una realidad? Y si lo es ¿es una realidad tal cual es aquel objeto de estudio por parte de la ciencia como la define Bunge (2011a; 2011b; 2014)? ¿o qué tipo de realidad es?

Las respuestas son diversas, según incluso la concepción filosófica del Derecho que se tenga. Para Kelsen, por ejemplo, la realidad jurídica -tal como se ha explicado en el apartado anterior- es de tipo normativa, por imputación. Concepto según el cual no podría identificarse a esa realidad jurídica con la realidad que se exige como contenido del lenguaje-objeto en la teoría de Tarski (2005), a razón de que la realidad jurídica es una realidad creada -y en constante creación y cambio- por arte y no por naturaleza -empleando términos aristotélicos.

Por otro lado, autores como Michael Moore (1982; 1992) o Ronald Dworkin (1989; 2012; 2013) sí defenderían la existencia de una realidad jurídica incluso a nivel metafísico, independiente del conocimiento y voluntad humana, que sí podría hacer posible la verificación (o falsación) de las proposiciones u oraciones enunciativas -esto es, de lo que se dice acerca de la realidad jurídica. Sin embargo, el problema del que adolecen las concepciones tales como 
la de Moore o la de Dworkin se encuentra en la existencia de dicha realidad jurídica. Pues el acceso, a la misma, como lo diría Dworkin, requiere de un esfuerzo sobrehumano, capacidad que la tiene su “Juez Hércules", pero de la que incluso carecía el mismo Dworkin al no poder dar respuesta sobre dicha realidad.

Tanto la falibilidad de los órganos productores de leyes, como de aquellos encargados de su aplicación, sumado al empleo de lenguaje natural y la visión histórica de lo que ha significado Derecho a lo largo del tiempo, hacen insatisfactoria la tesis de Dworkin de que, en el Derecho, y para cada caso, exista una única respuesta correcta derivada de aquel mundo jurídico superior al cual sólo puede accederse con una capacidad sobrehumana. De ahí que para Alessio Sardo (2013) diría que aquella objetividad pretendida por Alexy es más "un acto de fe".

Un concepto reputado como realidad, cuya esencia es inobservable humanamente no podría considerarse propiamente como una realidad (cfr. Bunge, 2011a; 2011b; 2014). Esta falencia, buscaría ser superada por la concepción de Alexy (1991), para quien, si bien existen principios en el Derecho, el contenido de los mismos no proviene de una "iluminación" por parte de un "mundo superior", sino de un discurso racional -aunque tales principios tengan una base moral, además de aquella jurídica.

Finalmente, sea que se repute como única realidad jurídica a una realidad normativa formada por imputación y no causalidad (Kelsen, 1982), o bien a una formada por tanto por imputación como por un discurso racional sobre una base moral crítica (Alexy, 1991), en ambos casos se trata de artificios. La diferencia radica en qué marca el límite de discreción para dichos artificios, si en aquel producto institucionalizado llamado normas jurídicas positivas, o si, por otro lado, trasciende hasta una zona de mayor penumbra, pero también de mayores posibilidades a través de los principios jurídicos.

En tal sentido, partiendo de lo propuesto por Tarski (1956; 2005), no es plausible afirmar una noción de verdad en el Derecho, pese a poder afirmar que la ciencia jurídica -o no tan ciencia, como se ha visto en Bunge- sea un metalenguaje del Derecho. Pues la denominada realidad jurídica, no es identificable con la realidad científica a la que dedican sus obras tanto Bunge (2011a; 2011b; 2014) como Tarski (1956; 2005).

\section{La verdad según el falsacionismo de Popper}

Tarski no sería el único pensador en pretender definir la verdad. De alguna manera, motivado por los trabajos de Tarski, Karl Popper (1980) también buscaría lo mismo, aunque propiamente no una definición satisfactoria de la verdad -o por lo menos, no en el mismo sentido que Tarski- sino una aproximación a la misma.

Para Popper (1991; 1995) el conocimiento es conjetural. Existen tendencias conscientes o inconscientes en las personas que orientan lo que consideran como verdadero. Este sería un elemento subjetivo que afectaría la investigación en la ciencia. Así, para Popper, ciencia será aquella que pueda resistir refutaciones, pues confirmar una tendencia a través de elementos del pasado es posible, pero ello sólo nos apartaría de la búsqueda de lo verdadero: pues ello cristaliza el conocimiento. Lo contrario, para Popper (1980), será meramente una seudociencia.

No yerra entonces Ochoa Disselkoen (2007) al calificar la epistemología de Popper como evolucionista. Ello en el sentido de que para Popper (1980) el conocimiento implicaba probabilidad 
y contingencia. La aproximación a la verdad se produce según aquello que sea más probable en un tiempo y lugar determinado, lo que implica -a su vez- la admisión de cambios en lo que se considera verdadero, según el surgimiento de nueva evidencia que refute o confirme lo probablemente verdadero en dicho tiempo y lugar -de ahí la implicación de contingencia necesaria.

Esto lleva a considerar a Popper que frente a la verdad sólo tenemos aproximaciones contingentes, aunque las refutaciones al conocimiento presente parezcan suponer avances en el caminar hacia lo verdadero. De ahí que Ochoa Disselkoen (2007) concluye razonablemente en la separación de Popper del pensamiento de Tarski, y el acercamiento del mismo Popper a un pensamiento pragmático de la verdad.

Considerando ello, nuevamente se propone cuestionar la aplicabilidad o pertinencia de la teoría de Popper en el Derecho. No enfocándonos a aspectos de razonamiento probatorio o acerca de la existencia o vigencia de formulaciones normativas (Lariguet, 2002), sino en cuanto a la validación -o falsación como diría Popper- de las proposiciones normativas, esto es, de lo que se dice que es el Derecho vigente.

La respuesta, desde Popper, es que no. Tal como lo indica el mismo Karl Popper, quien sólo admite "un sistema entre los científicos o empíricos si es susceptible de ser contrastado por la experiencia" (Popper, 1980, p. 40). Así, siendo que las formulaciones normativas se expresan en la forma de enunciados deónticos, no podrían considerarse como empíricos. Ergo, no podría contrastarse si determinada proposición normativa se adecua o no en forma idónea con la realidad normativa, puesto que se trata de una "realidad" no contrastable empíricamente -pues según el propio Alexy (2013) dicha "realidad" estaría justificada únicamente en el plano metafísico.

Sea bajo un enfoque positivista del Derecho, o bien sea bajo un enfoque principialista del mismo, conforme se ha argumentado en el punto anterior relativo a Tarski. Aun admitiendo la posibilidad de que la moral racional, sea una materia vinculada al Derecho, y los principios jurídicos el medio para concretizarla, la verificación (o falsación) del contenido de tales principios tampoco podría ser contrastable empíricamente, pues dicho contenido es finalmente imputado según la cosmovisión actual del intérprete, y no según una percepción contrastable empíricamente -al no tenerse un objeto con el cual contrastar, como señala Guastini (2014).

Esto nos lleva a la misma aseveración antes realizada: no es plausible afirmar una noción de verdad en el Derecho, aún incluso desde una perspectiva pragmática como la que finalmente aporta Popper (1980; 1991; 1995).

\section{Crisis y revolución en Kuhn}

Aun manteniendo una perspectiva evolucionista de la ciencia, Thomas Kuhn se distinguiría de Popper, pues para Kuhn, la falsación como elemento de aproximación a la verdad según Popper, no podría explicar satisfactoriamente todos los fenómenos y problemas suscitados en un tiempo y lugar determinados -dudando así incluso de la existencia de la falsación como elemento de avance de la ciencia (Kuhn, 2004).

Para Kuhn (2004) el avance de la ciencia era considerado como un proceso discontinuo afectado por anomalías, crisis y revoluciones que propician el cambio y establecimiento de paradigmas. Esto, para Kuhn permite considerar a la ciencia como una ciencia madura en el sentido de presentar una ciencia que puede responder a los problemas de su tiempo. 
Los paradigmas son, entonces, un concepto central en la teoría de Kuhn (2004), quien los considera como un modelo o patrón aceptado que comparte una comunidad científica, y cuya existencia establece el problema que debe resolverse. El surgimiento de un paradigma implica el otorgamiento de dicho status a una teoría científica que explica o responde a una realidad presente en un tiempo y espacio determinados. Y el rechazo de un paradigma, a su vez, implica simultáneamente la aceptación de otro que explique o responda mejor a la realidad presente. Esto último implicaría la invalidación de la teoría científica a la cual se consideró en su momento dicho status de paradigma, ello por un juicio comparativo con otra teoría -la que, de obtener reconocimiento adquiriría entonces ahora dicho status. A esto llamará Kuhn (2004) como revolución científica.

Respecto a esta teoría formulada, Carl G. Hempel le plantearía una pregunta a Kuhn acerca de si este reconocía o no la diferencia entre explicar la elección o reconocimiento de una teoría, y justificar dicho comportamiento. Pregunta que sería respondida por Kuhn en un simposio sobre la filosofía de Hempel (Kuhn, 2002). Según Kuhn, sí existía diferencia entre explicar y justificar la elección teórica. El aspecto explicativo se enfocaba en la capacidad de determinada teoría para explicar una realidad determinada o para responder a los problemas de la misma. Y el aspecto justificativo se enfocaba en criterios no arbitrarios, al pertenecer al conjunto de disciplinas de contenido empírico.

Partiendo de lo anotado ¿sería posible hablar de paradigmas en el Derecho? Aunque Kuhn (2004) toma la palabra paradigma del lenguaje común "a falta de otro término mejor" (p. 51) que le permitiese explicar su teoría, desde el pensamiento de Kuhn no sería posible hablar de paradigmas científicos en el Derecho. Ello debido a que para Kuhn (2002) el Derecho no reúne condiciones necesarias y suficientes para la pertenencia de dicha disciplina al campo de la ciencia. Desde un punto de vista explicativo, el Derecho no tendría una racionalidad mínima para estas condiciones al carecer por sí mismo de contenido empírico para validar una teoría como paradigma. Y desde el punto de vista justificativo las teorías en el Derecho tampoco cumplirían con las condiciones mínimas al establecerse propiamente bajo criterios directamente arbitrarios: basta ver el ejemplo de las normas, cuyo contenido es determinado bajo un margen de discrecionalidad, para unos delimitado por reglas primeras y secundarias (Raz, 1986; Hart, 2009), y para otros además (por un campo que parece extender dicha discreción hasta lo indeterminado) por principios de contenido axiológico (Alexy, 1991; 1993; 2002; 2013). Una aceptación extendida, entonces, de una teoría del Derecho, o incluso de una interpretación determinada, obedece más a fenómenos sociológicos (Bourdieu \& Teubner, 2002) y culturales (Kahn, 2001), y no propiamente a criterios científicos como los concibe Kuhn (2002; 2004).

\section{Infames reflexiones finales}

¿Existe una relación entre epistemología y Derecho? Sí, al menos una relación indirecta e instrumental, pues una disciplina puede servirse de la otra, aunque no para sus objetivos centrales.

Tal como se ha argumentado, ninguno de los cuatro autores, centrales en la historia contemporánea de la ciencia, acepta que el Derecho consiga las condiciones mínimas para su consideración como ciencia. La verificabilidad, el falsacionismo, o la aceptabilidad racional -según argumentan Bunge, Tarski, Popper y Kuhn- requieren de una posibilidad de contrastación empírica y de no-arbitrariedad que no puede explicar el Derecho. 
La verdad importa, e importe mucho, para el Derecho. Su relevancia es plenamente visible como fuente material para la justificación fáctica en la dación de leyes, así como en materia probatoria. Sin embargo, ello es discutible respecto a las formulaciones normativas, ¿existirá un mundo jurídico como el que concebía Dworkin? ¿o un método que nos permita acceder al campo de la moral objetiva como parece pretender Alexy? ¿será posible responder estas preguntas desde una epistemología jurídica? Al menos desde el pensamiento de los cuatro científicos y filósofos comentados, no. No es posible ni la concepción de una "epistemología jurídica", ni tampoco de una racionalidad interna objetiva en el Derecho. Lo que limita la posibilidad de sostener ello es que el Derecho no preexiste al ser humano, el Derecho que construyen las autoridades y aplican los tribunales no es ni natural ni divino, los operadores de Derecho no describen una realidad existente, sino que prescriben cómo debería ser dicha realidad, y no hay modo de confrontar empíricamente tal contenido prescriptivo o normativo con cierta "realidad superior" o esquema alguno que exista en un "mundo ideal".

Ello no descalifica de ninguna manera la trascendencia del Derecho, pues se habla de dos cosas diferentes. El contenido del Derecho se enfoca en cuestiones deontológicas, y no en cuestiones ontológicas -aunque esto último es imprescindible para poder sustentar aquel debe ser que se traducirá en norma jurídica. El Derecho, entonces, hace aprovechamiento de la ciencia, y la ciencia, también aprovecha al Derecho para su desarrollo óptimo -y así evitar indeseables experiencias como las dejadas por los regímenes totalitarios que restringieron el desarrollo de la ciencia.

Sin embargo, el lector podría preguntarse ¿cuál es la relevancia de este asunto? ¿para qué distinguir entre ciencia - propiamente dicha- y Derecho? Y la respuesta sería: para no extrapolar. El Derecho, sin duda, requiere de un discurso racional para su desarrollo, pero dicho discurso no puede tener como objeto alcanzar la contemplación de una verdad jurídica, sino conducir la discrecionalidad de sus operadores. El Derecho es pues, un instrumento, un instrumento necesario para la supervivencia y el desarrollo de todas aquellas actividades que puede pretender el ser humano al efecto, dentro de las cuales se encuentra la ciencia. 
Referencias bibliográficas

Alexy, R. (1991). Teoría de la Argumentación Jurídica. La teoría del discurso racional como teoría de la fundamentación jurídica. (M. Atienza, \& I. Espejo, Trads.) Madrid: Centro de Estudios Constitucionales.

Alexy, R. (1993). Teoría de los derechos fundamentales. Madrid: Centro de Estudios Políticos y Constitucionales.

Alexy, R. (2002). Epílogo a la teoría de los derechos fundamentales. Revista Española de Derecho Constitucional, año 22, núm. 66, 13-64.

Alexy, R. (2013). Derecho, moral y la existencia de los derechos humanos. Signos filosóficos, 15(30), 153-171.

Anscombe, G. E. (1991). Intención. (A. I. Stellino, Trad.) Barcelona: Paidós.

Bourdieu, P., \& Teubner, G. (2002). La fuerza del Derecho. Bogotá: Siglo del Hombre Editores.

Bulygin. (2018). Lógica deóntica, normas y proposiciones normativas. (P. E. Navarro, J. L. Rodríguez, \& G. B. Ratti, Edits.) Madrid: Marcial Pons.

Bunge, M. (1976). ¿Qué es y para qué sirve la epistemología? Revista de la Univeridad de México, $2,1-7$.

Bunge, M. (2011a). Las ciencias sociales en discusión: una perspectiva filosófica. (H. Pons, Trad.) Editorial Sudamericana: Buenos Aires.

Bunge, M. (2011b). Las pseudociencias jvaya timo! Barcelona: Laetoli.

Bunge, M. (2014). La ciencia. Su método y su filosofía. Barcelona: Debolsillo.

Ceberio, M., \& Watzlawick, P. (1998). La Construcción del Universo. Barcelona: Herder.

Dworkin, R. (1989). Los derechos en serio. Barcelona: Ariel.

Dworkin, R. (2012). El Imperio de la Justicia. (C. Ferrari, Trad.) Barcelona: Editorial Gedisa.

Dworkin, R. (2013). Una cuestión de principios. (V. Boschiroli, Trad.) Buenos Aires: Siglo XXI Editora Iberoamericana.

Guastini, R. (2014). Interpretar y argumentar. Madrid: Centro de Estudios Políticos y Constitucionales.

Hart, H. L. (2009). El concepto de derecho. (G. Carrió, Trad.) Buenos Aires: Abeledo Perrot.

Hospers, J. (1984). Introducción al análisis filosófico. (J. C. Armero, Trad.) Madrid: Alianza Editorial.

Kahn, P. W. (2001). El análisis cultural del Derecho. Una reconstrucción de los estudios jurídicos. Barcelona: Gedisa.

Kelsen, H. (1982). Teoría Pura del Derecho (Segunda ed.). (R. J. Vernengo, Trad.) México D. F.: Universidad Nacional Autónoma de México.

Kuhn, T. (2002). Racionalidad y elección de teorías. En T. Kuhn, El camino desde la estructura. Ensayos filosóficos, 1970-1993, con una entrevista autobiográfica (A. Beltrán, \& J. Romo, Trads., pp. 247-255). Barcelona: Paidós.

Kuhn, T. (2004). La estructura de las revoluciones científicas. (A. Contin, Trad.) México: Fondo de Cultura Económica.

Lariguet, G. (2002). La aplicabilidad del programa falsacionista de Popper a la ciencia jurídica. Isonomía, 17, 183-202.

Moore, M. (1982). Moral reality. Wisconsin Law Review, 1061-1156.

Moore, M. (1992). Moral Reality Revisited. Michigan Law Review, 90, 2423-2523.

Nino, C. S. (1992). Un país al margen de la ley. Emecé: Buenos Aires. 
Ochoa Disselkoen, H. (2007). Popper y el concepto de verdad. Filosofía UIS, 6, 179-190.

Paulson, S. L. (2000). Fundamentación crítica de la doctrina de Hans Kelsen. Bogotá: Universidad Externado de Colombia.

Popper, K. (1980). La lógica de la investigación científica. (V. Sánchez de Zalava, Trad.) Madrid: Tecnos.

Popper, K. (1991). Conjeturas y refutaciones. El desarrollo del conocimiento científico. (N. Miguez, Trad.) Barcelona: Paidós.

Popper, K. (1995). La responsabilidad de vivir. Escritos sobre política, historia y conocimiento. Barcelona: Paidós.

Raz, J. (1986). La autoridad del Derecho. (R. Tamayo, Trad.) México: Universidad Nacional Autónoma de México.

Sardo, A. (2013). Teorías de la ponderación. Análisis crítico. En P. Grández, \& F. Morales, La argumentación jurídica en el estado constitucional (pp. 239-272). Lima: Palestra Editores.

Störig, H. J. (2012). Historia universal de la Filosofía (Segunda ed.). (A. Gómez Ramos, Trad.) Madrid: Tecnos.

Tarski, A. (1956). Logic, Semantics, Metamathematics: Papers from 1923 to 1938. (J. H. Woodger, Trans.) Oxford: Clarendon Press.

Tarski, A. (2005). La concepcíon semántica de la verdad y los fundamentos de la semántica. En L. Valdes Villanueva, La búsqueda del significado (M. Bunge, E. O. Colombo, E. Arias, \& L. Fornasari, Trads., pp. 299-335). Madrid: Tecnos 